\title{
Interferenz bei Referenz im Übersetzungsprozess
}

\author{
Gyde Hansen \\ Copenhagen Business School (CBS)
}

Reference with noun phrases can only be described by drawing on different branches of linguistics such as pragmatics, semantics, syntax, morphology, phonology and stylistics. In a contrastive analysis of the use of articles in pairs of languages such as Danish and German, it has been shown to be expedient to deal first of all with the material in terms of a common pragmatic and text-semantic tertium comparationis (i.e. comparative framework) and subsequently to include constraints from other levels of description. Although Danish and German are in fact closely related languages, they differ considerably with respect to reference and the use of articles, and these - at times quite complicated - differences lead to a great deal of interference in translation. In a series of experiments in which translation processes were studied using the computer program Translog (developed by Arnt Lykke Jakobsen of the Copenhagen Business School), it has been shown that an effective means of combatting reference-related interference relating to reference in translation processes is to give individual consultation, through which the information obtained from observations made during the translation process and the individual strengths and weaknesses of translators can be combined with the insights of contrastive linguistics.

\section{Einleitung}

Interferenz bei unmittelbarem Sprachkontakt, wie es das Übersetzen ist, ist nicht zu vermeiden, und schon gar nicht, wenn zwischen so verwandten Sprachen übersetzt wird, wie dem Dänischen und dem Deutschen. Juhász (1970:9ff.) definiert Interferenz linguistisch als "die durch die Beeinflussung von anderen sprachlichen Elementen verursachte Verletzung einer sprachlichen Norm bzw. der Prozess der Beeinflussung." Er unterscheidet zwischen Interferenz der Fremdsprache auf die Muttersprache und Interferenz der Muttersprache auf die Fremdsprache, und er definiert sie der Art nach als phonetische, grammatische und semantische Interferenz. In der Übersetzungswissenschaft definiert Kupsch-Losereit (1998:167) Interferenz nicht nur linguistisch, sondern kommunikativ als "Projektion von Merkmalen des Ausgangstextes (AT) in den Zieltext (ZT), deren Ergebnis eine Verletzung der (parole-bezogenen) ZT-Normen, -Konventionen und -Diskurse ist, also lexikalischer, thematisch-inhaltlicher, mikro- und makrostruktureller, situativer sowie kultureller Art sein kann."

Interferenzprobleme treten bei Übersetzungen zwischen dem Dänischen und Deutschen in beiden Übersetzungsrichtungen auf allen Ebenen der Sprachbeschreibung auf, und zwar besonders auf der pragmatischen, der grammatischen und der lexikalischen Ebene. Oft sind mehrere 
Ebenen gleichzeitig betroffen. Niemand ist gegen diesen Fehlertyp gefeit. Bilinguale Übersetzerinnen und Übersetzer, die Deutsch nicht erst in der Schule und an der CBS gelernt haben, sondern auch schon in ihrer Familie oder durch lange Aufenthalte in Deutschland, scheinen solchen Fehlern gegenüber ebenso anfällig oder noch anfälliger zu sein, als die Übersetzerinnen und Übersetzer, die Deutsch erst als zweite Fremdsprache in dänischen Schulen und an der CBS erworben haben.

Obwohl es manchmal so aussieht, als sei die Leistung der kontrastiven Linguistik für das Übersetzen in der Praxis begrenzt (Snell-Hornby 1986:20), ist ihr Nutzen für das Übersetzen unumstritten (Wilss 1994:18; Hansen 1997; Schreiber in Druck). Dies gilt besonders, wenn es sich um die kontrastive Textlinguistik handelt, die auch die Bedingungen der Kommunikationssituation berücksichtigt (Hansen 1995:2). Die Fragen, die hier untersucht werden sollen, sind:

1. Was könnte die kontrastive Linguistik zur Vermeidung von Fehlern, wie z. B. den so häufigen Interferenzen leisten?

2. Bei welchen Übersetzungsproblemen liegt der größte Bedarf an Regeln vor, und wie lässt sich das feststellen?

3. Wie sollten solche unmittelbar anwendbaren, d.h. benutzerfreundlichen Gebrauchsregeln aussehen?

Diese Fragen werden hier am Beispiel der Interferenz beim Übersetzen aus der Muttersprache in die Fremdsprache untersucht und zwar was die Referenz auf Gegenstände oder Sachverhalte mit Hilfe von Nominalphrasen betrifft. Unter Referenz verstehe ich dabei nicht nur die aus der Semasiologie und der Onomasiologie bekannte, eher statische Beziehung zwischen der Nominalphrase und dem Gegenstand oder Sachverhalt der außersprachlichen Welt, wie sie oft an Dreiecks- oder Trapezmodellen dargestellt worden ist (Ogden \& Richards 1948:11; Heger 1971:26), sondern dynamisch die Handlung in einer Kommunikationssituation, zu der ein Sender ein Zeichen benutzt. Dazu stütze ich mich auf Strawson 1950:326ff, der sagt:

"Mentioning" or "referring", is not something an expression does; it is something that someone can use an expression to do. Mentioning, or referring to something is a characteristic of a use of an expression just as "being about" something and truth or falsity, are characteristics of a use of a sentence.

Er betont dabei auch die Notwendigkeit des Kontextes für die Referenz:

The context of an utterance is of an importance, which it is almost impossible to exaggerate; and by "context" I mean at least the time, the place, the situation, the identity of the speaker, the subjects which form the immediate focus of interest, and the personal histories of both the speaker and those he is addressing. Besides context there is of course, convention - linguistic convention.

Die verschiedenen Referenztheorien kann ich in diesem Zusammenhang nicht diskutieren. Für die kontrastive Analyse des Artikelgebrauchs im 
Dänischen und Deutschen hat es sich als nützlich erwiesen, davon auszugehen, dass beim Gebrauch aller Nominalphrasen von Referenz die Rede sein kann. Zum Problem der referierenden bzw. nicht-referierenden Nominalphrasen siehe Hansen (1986:35) und Hansen (1998:16ff.).

Die dänischen Nominalphrasen, von denen hier die Rede sein wird, bestehen aus einem Nomen und einem appositionellen Attribut oder aus einem Kompositum. Es ist jeweils ein Eigenname enthalten. Sie stammen alle aus einer Broschüre über die dänische Stadt Skagen und ihre Umgebung, an der Nordspitze Jütlands. Der Text wurde an der CBS in einem Kurs über Übersetzungsprozesse dazu benutzt, festzustellen, auf welchen Gebieten die Studierenden besonders große Schwierigkeiten haben, und wie man diese in einem Prozess der Aktionsforschung erkennen und bewältigen kann. Die Versuchspersonen waren 6 Studierende im zweiten Teil ihres Studiums zum "staatlich geprüften Übersetzer und Dolmetscher", die ich im Folgenden als die Versuchspersonen EN, TO, TRE, FIRE, FEM und SEKS bezeichnen werde. Die Versuchspersonen TO und FIRE sind bilingual. Der Text enthält viele andere Übersetzungsprobleme und wurde nicht speziell aufgrund der auftretenden Interferenzprobleme gewählt. Diese erkannten wir im Laufe des Prozesses. Der AT und der Übersetzungsauftrag sind als Anhang abgedruckt.

\section{Methode}

\subsection{Schritte der Untersuchung}

Zur Feststellung, wo der größte Bedarf an Unterstützung durch u. a. kontrastive Gebrauchsregeln herrscht, hat sich das Übersetzen desselben Textes zuerst spontan und unter Zeitdruck und dann noch einmal ohne Zeitdruck und mit allen Hilfsmitteln, d.h. Wörterbuch, Internet und evtl. Telefon, als geeignete Methode bewährt (Hansen 2002).

Dadurch, dass man als Beobachter aufgrund der Übersetzung mit Zeitdruck (ÜmZ) die spontane, automatische Leistungsfähigkeit unter extremen Umständen registrieren kann, und mit der Übersetzung ohne Zeitdruck (ÜoZ) die Leistungsfähigkeit unter "normalen", friedlicheren Arbeitsbedingungen, bekommt man einen Einblick in die Spannweite der Kompetenz der Versuchspersonen. Man testet auf diese Weise, wie sich bei den Versuchspersonen die schwächste und die höchste Leistungsfähigkeit zueinander verhalten. Bei Zeitdruck treten Unsicherheiten auf, die dann verschwinden können, wenn Zeit genug vorhanden ist. An dem Unterschied, der zwischen den beiden Übersetzungen besteht, lässt sich erkennen, inwieweit die Übersetzerinnen und Übersetzer überhaupt Probleme haben, ob sie auf ihre Probleme aufmerksam werden, und über welche Methoden, Regeln und Strategien sie verfügen, um erkannte Übersetzungsprobleme zu lösen. Versuche (Hansen 2002) haben gezeigt, dass Versuchspersonen, die an problematischen Stellen auf Methoden und Regeln zurückgreifen konnten, sich verbesserten, sobald sie Zeit genug hatten, wohingegen bei denjenigen, die 
keine oder kaum Methoden und Regeln kannten, zwischen den beiden Zieltexten eine große Übereinstimmung bestand. Übereinstimmung zwischen den beiden Leistungen gibt es auch bei Versuchspersonen, die in keiner der beiden Arbeitssituationen Probleme hatten.

Die Versuchspersonen bekamen die Möglichkeit, ihren Zieltext der ÜoZ im Papierausdruck zu korrigieren. Die Zeit, die sie dazu benötigten, benutzte ich dazu, beide Zieltexte zu korrigieren und festzustellen, wo die Unterschiede und Übereinstimmungen zwischen den Produkten der ÜmZ und der ÜoZ lagen. Diese Beobachtungen bildeten die Grundlage für eine Hypothese über die Kompetenz der Versuchspersonen und die Schwierigkeiten, die sie hatten. Unmittelbar nach der ÜoZ, die mit der Korrektur des Papierausdrucks abgeschlossen worden war, bat ich die Versuchspersonen ihren Übersetzungsprozess in einer Retrospektion mit Replay (siehe 1.2.) zu kommentieren. Während der Prozess vor uns auf dem Computerschirm ablief, konnte ich feststellen, welche Textstellen, Probleme und Pausen sie unaufgefordert bemerkten und kommentierten. An den Bewegungen während des Prozesses, die alle auch in der Log Datei (siehe 1.2.) abzulesen sind, erkennt man u.a. auch den Grad ihrer Unsicherheit.

Meine Hypothesen über die Kompetenz der Versuchspersonen benutzte ich nach der Retrospektion mit Replay als Ausgangspunkt für offene Fragen und ein klärendes Interview. Ich hatte dazu im Zieltext der ÜoZ sowohl gute als auch fehlerhafte Übersetzungsvorschläge unterstrichen, und bat die Versuchspersonen um Kommentare zu diesen Textstellen.

Die Versuchspersonen und ich arbeiteten nun an einem Veränderungsprozess mit dem Ziel, dass sie sich ihrer Probleme bewusst werden, dass sie sie selber formulieren und dass sie sie schließlich auch selber lösen. Durch diese aktive Zusammenarbeit bekam ich wiederum neue Erkenntnisse darüber, inwieweit die Versuchspersonen ihre Probleme erkennen und verstehen, und welche Aspekte in einem Problemkomplex es sind, die ihnen besonders Schwierigkeiten bereiten.

Bei der Analyse der Ergebnisse untersuchte ich mit Hilfe der Aufzeichnungen der Log Datei von Translog außerdem den Zeitverbrauch der Versuchspersonen an den problematischen Textstellen, denn auch dieser kann ein Zeichen dafür sein, dass die Versuchspersonen über ein Problem nachgedacht haben.

\subsection{Zeitdruck und Retrospektion}

Um die Versuchspersonen unter Zeitdruck zu setzen, benutzte ich Translog 2000, die letzte Ausgabe einer Computer Software zur Registrierung von Schreibprozessen, die an der Copenhagen Business School von Arnt Lykke Jakobsen entwickelt wurde (Jakobsen 1999a, 1999b). Über Translog kann man bestimmen, wie der Ausgangstext präsentiert wird. Der Text kann einerseits in seiner Gesamtheit erscheinen, andererseits aber auch in definierten Abschnitten oder in Sätzen. Es können auch verschiedene zeitliche Bedingungen eingegeben werden. Entweder kann man die Versuchs- 
person selber bestimmen lassen, wie lange der Text oder Textabschnitt auf dem Bildschirm stehen bleiben soll, oder es ist möglich, Beschränkungen einzubauen und den Text oder die Textabschnitte nach einem bestimmten Zeitraum matt werden oder verschwinden zu lassen. Wenn es sich um Abschnitte handelt, ist der erste Abschnitt sofort zu sehen, während weitere Abschnitte nach einer vorher festgelegten Anzahl von Sekunden im oberen Teil des Bildschirms erscheinen und dann wieder verschwinden. Die Prozessdaten können nach dem Versuch durch eine "replay facility" zu einer dynamischen Wiedergabe des Schreibprozesses abgerufen werden, eine Funktion, die sich besonders zur Konfrontation der Versuchsperson mit ihrem eigenen Schreibprozess eignet (Retrospektion mit Replay). Außerdem zeigt eine "view facility" eine Momentaufnahme der Log Datei. Sie dokumentiert den gesamten Schreibprozess. Bestimmte Kodes dienen hierbei als Zeichen für Pausen, und es werden sämtliche Anschläge und Cursorbewegungen registriert, so dass man jede Bewegung im Text und damit auch alle Korrekturen während des Prozesses erkennen kann. Die Pausenlänge wird mit einer Genauigkeit von 10 Millisekunden angegeben. Der geschriebene Text kann ganz normal ausgedruckt werden, so dass man das Produkt vor Augen hat.

Da ich die Leistung bei den automatischen Prozessen feststellen wollte, sollte die Zeitvorgabe individuell so berechnet sein, dass jede Versuchsperson genau genug Zeit hatte, eine Textstelle spontan zu übersetzen, aber nicht so viel, dass sie etwas ändern konnte. Mein Kriterium für die Richtigkeit der Zeitvorgabe war, dass bei allen Versuchen mit Zeitdruck an wenigstens einer Stelle im Text, z. B. durch Reduktion oder eine Auslassung deutlich $\mathrm{zu}$ sehen sein sollte, dass die Versuchsperson wirklich unter Zeitdruck gewesen war.

Den Zeitdruck habe ich folgendermaßen errechnet. Ich ließ die Versuchspersonen mehrere Übersetzungen zu Hause, d.h. unter gewohnten Verhältnissen, mit Translog anfertigen und berechnete aus den Zeitintervallen, die sie zur Übersetzung von 10 Zeilen von unterschiedlichem Schwierigkeitsgrad brauchten, und bei denen sie nicht im Wörterbuch nachgeschlagen hatten, ihre durchschnittliche Übersetzungsgeschwindigkeit. Sie bekamen dann bei der Übersetzung in die Muttersprache noch 40 Sekunden und bei der Übersetzung in die Fremdsprache noch 50 Sekunden Denkzeit hinzu. Diese Werte hatte ich aus vorbereitenden Versuchen mit verschiedenen Versuchspersonen abgeleitet.

\section{Kontrastive linguistische Aspekte}

Die Interferenzfehler, die hier gemacht werden, sind besonders dadurch interessant, dass sie gleichzeitig mehrere kontrastive linguistische Aspekte betreffen - einerseits die Struktur der Nominalphrase, andererseits den Artikelgebrauch und manchmal auch die Wortwahl. Es handelt sich also um ein komplexes Übersetzungsproblem. Einer der beschriebenen Fehler entsteht nur indirekt durch Interferenz, denn es scheint sich um eine Interfe- 
renzphobie zu handeln (Kussmaul 1995:19). Die Struktur der Nominalphrasen und der Artikelgebrauch werden in diesem Abschnitt weiter erläutert.

\subsection{Struktur der Nominalphrasen}

\subsubsection{Im Ausgangstext}

Bei folgenden Nominalphrasen traten Interferenzen oder Interferenzphobie im Zieltext auf:

1. Skagen by (die Stadt Skagen)

2. Skagen-området (das Gebiet Skagen)

3. Skagen Festival (das Skagen Festival)

4. Skagbo (in Skagen einheimisch)

5. St. Hans aften/Sankthansaften (das Johannisfeuer zur Mittsommernacht)

Bei Skagen by, Skagen Festival und St. Hans aften handelt es sich um durch appositionelle Attribute erweiterte Nominalphrasen. (Zu den Bezeichnungen "appositionelles Attribut" oder "enge Apposition" siehe Fabricius-Hansen \& von Stechow 1989:176ff.). Skagen-området ist als Kompositum zu betrachten. Auch von St. Hans aften gibt es ein Kompositum: Sankthansaften. Bei Skagbo ist von einem Kompositum aus Skagen und beboer die Rede. Analog dazu gibt es z. B. auch Hvenbo, d.i. ein auf der Insel Hven Einheimischer. Im dänischen AT wird die Konstruktion als Prädikatsnomen benutzt, aber sie tritt sonst auch in anderen Funktionen der Nominalphrase auf, u.a. als Präpositionalattribut oder wiederum als Teil eines Kompositums; Beispiele:

(1) 44 procent af skagboerne svarer, at der efter deres mening er for mange turister i byen. (Morgenavisen Jyllands-Posten, 30.06.2002: Brunch, 4)

(44 Prozent der einheimischen Skagener antworten, dass es ihrer Meinung nach zu viele Touristen in der Stadt gibt.)

(2) Ingen skagbobørn leger på gaden. (Morgenavisen Jyllands-Posten, 30.06.2002: 1. Sektion, 9)

(Auf der Straße spielen keine einheimischen Skagener Kinder.)

\subsection{2. Übersetzungsmöglichkeiten in der Zielsprache Deutsch}

Es werden hier, der Definition von Referenz als Handlung in einem Kontext entsprechend, nur die Konstruktionen angegeben, die auch pragmatisch und semantisch in den Kontext passen würden.

1. Skagen by: Die beste Konstruktion ist hier ein Nomen mit Apposition: "Die Stadt Skagen", wobei sich aber die Reihenfolge der Glieder im Verhältnis zum Dänischen ändert. 
2. Skagen-området: Es ist wichtig, dass sich die Nominalphrase im Dänischen sowohl auf die Stadt als auch auf die Umgebung bezieht und nicht nur auf die Umgebung von Skagen. Daher gibt es nach dem Kontext vier Möglichkeiten: "Das Gebiet Skagen", "Das Gebiet von Skagen", "Skagen und Umgebung" oder einfach "Skagen". Im ersten Fall handelt es sich um eine Konstruktion mit Nomen und Apposition, im zweiten Fall um ein Nomen mit einem Präpositionalattribut, im dritten Fall um zwei nebengeordnete Nomina und schließlich um eine Reduktion auf ein einfaches Nomen, den Namen der Stadt. Konstruktionen wie "die Umgebung von Skagen" oder "die Skagener Umgebung" sind sowohl Interferenzfehler als auch semantisch problematisch, denn die Stadt selbst wäre damit ausgeschlossen, was im Textzusammenhang überhaupt nicht der Intention des Senders der Broschüre entspricht. Eine Bezeichnung wie "Raum Skagen", der im ZT der Broschüre benutzt wurde, wird von den potentiellen deutschen Zieltextempfängern, die ich gefragt habe, als "geographisch-politisch" aufgefasst.

3. den årlige Skagen Festival: Da es sich hier in beiden Sprachen um eine Konstruktion mit Nomen und appositionellem Attribut handelt und weil die NP insgesamt ein Eigenname ist, bleibt die Konstruktion im Deutschen gleich "das jährliche Skagen Festival". Analog dazu spricht man im Deutschen auch von "Roskilde Festival" und "Schleswig-Holstein Musikfestival". Bei diesem Beispiel besteht die Gefahr von Interferenzphobie, denn in den Fällen, wo nicht die ganze NP als ein Eigenname aufgefasst wird, kann man im Deutschen nicht die gleiche Konstruktion benutzen. Beispielsweise müsste: I London Zoologiske Have mit "Im Londoner Zoo" und nicht mit "Im London Zoo" übersetzt werden, und Skagen Ravmuseum wäre auf Deutsch "das Skagener Bernsteinmuseum" oder "das Bernsteinmuseum von Skagen" und nicht "das Skagen Bernsteinmuseum". Die Übersetzung "das alljährliche Skagener Festival" entsteht also wahrscheinlich aus Angst vor Interferenz.

4. Skagbo: Es gibt im Kontext, wo die Rede von der Malerin Anna Ancher ist, mehrere Möglichkeiten: Nomen: "Skagnerin", Adjektiv + Nomen: "gebürtige/einheimische Skagnerin", oder eine Umschreibung: "stammte aus Skagen", "war in Skagen geboren und aufgewachsen".

5. St. Hans aften/Sankthansaften: Es handelt sich hier um den "Johannisabend" und das "Johannisfeuer", das zur Mittsommernacht angezündet wird. Der Begriff "Johannisfeuer" ist, obwohl er im Wörterbuch steht, in manchen deutschsprachigen Gebieten wenig gebräuchlich. Daher ist es pragmatisch notwendig, für den ZT-Empfänger eine Erklärung wie z. B. "zur Mittsommernacht" hinzuzufügen.

\subsection{Artikelgebrauch}

\subsubsection{Allgemein}

In diesem Zusammenhang können nur einige ganz generelle Regeln angegeben werden. 
Artikel bzw. Artikelwörter oder Artikelformen - es handelt sich um eine Gruppe von Artikeln und Pronomen (Mikkelsen 1911/2¹975; Hansen A. 1967; Diderichsen 1970; Vater 1963/21979) kommen in Kombination mit Substantiven vor, und mit diesen wird in einem Kommunikationsakt auf Gegenstände oder Sachverhalte referiert. Manchmal handelt es sich bei den Substantiven um Appellativa und manchmal um Propria.

Mit Hilfe der Artikel teilt der Sender dem Empfänger mit, wie dieser die Gegenstände oder Sachverhalte, auf die referiert wird und über die geschrieben oder gesprochen wird, in die Textwelt einordnen soll. Im Dänischen stehen ihm dazu u.a. der Schlussartikel, d.h. eine Flexionsendung, mit den Formen im Singular, commune: -en, Singular, neutrum: -et und Plural -ene zur Verfügung. Steht vor dem Nomen, das den Kern der Nominalphrase bildet, ein Adjektiv, benutzt man im Dänischen die vorangestellten Artikel und zwar im Singular, commune: den, im Singular neutrum: det und im Plural: de. Außerdem steht besonders bei Eigennamen, oft gar kein Artikel bzw. ein 0-Artikel ${ }^{1}$ vor dem Nomen.

Im Deutschen sind die bestimmten Artikel im Singular: der, die, das und im Plural: die, und besonders bei Eigennamen hat man den 0-Artikel.

Mit diesen Artikelformen signalisiert der Sender, dass er damit rechnet, dass der Empfänger die Gegenstände oder Sachverhalte identifizieren kann, und zwar entweder aufgrund seines Allgemeinwissens, oder aus der Situation heraus, oder weil sie schon vorher im Kontext erwähnt worden sind (Hansen 1986:53 und 1998:18).

Der Sender kann dem Empfänger aber auch mitteilen wollen, dass er auf einen im Text vorher nicht erwähnten, "neuen" Gegenstand referiert, über den er Nachinformation erwarten kann. Hierzu kann er im Dänischen die Formen des unbestimmten Artikels, en, et, O-Artikel und im Deutschen ein, eine, 0 -Artikel benutzen.

Beim Gebrauch von Nominalphrasen als Prädikativ ist, vorausgesetzt dass keine Identitätsrelation vorliegt, keine Nachinformation zu erwarten. Man benutzt, wenn besonderes Gewicht auf die Eigenschaften gelegt wird, die man einem Gegenstand oder Sachverhalt zusprechen möchte, en im Dänischen und ein im Deutschen, z. B.: Sie ist eine gute Lehrerin. Möchte man dagegen das Gewicht mehr auf die Zugehörigkeit zu der Klasse von Gegenständen legen, die unter einen Begriff fallen, benutzt man den 0 -Artikel in beiden Sprachen. Auch in Zweifelsfällen wird meistens der 0-Artikel benutzt, z. B.: Er war Arzt.

\subsubsection{Artikelgebrauch im dänischen Ausgangstext}

Betrachtet man die pragmatischen und textsemantischen Bedingungen zur Identifizierbarkeit der erwähnten Gegenstände oder Sachverhalte im Zusammenhang mit den Nominalphrasen im Ausgangstext, so ist bei Skagen by, Skagen-området und den årlige Skagen Festival zu bemerken, dass die Stadt Skagen schon mit der Überschrift der Broschüre "Lyset over Skagen" in die Textwelt eingeführt worden ist. Die Stadt ist aber auch aufgrund von 
Allgemeinwissen normalerweise identifizierbar. Der Skagener St. Hans aften (die Mittsommernacht) ist in Dänemark allgemein bekannt, und wird auch aufgrund eines berühmten, in der Broschüre abgebildeten Gemäldes des Skagener Malers P.S. Krøyer, aus dem Kontext heraus identifizierbar.

Bei allen diesen Nominalphrasen wäre also der Schlussartikel oder der bestimmte Artikel zu erwarten, mit dem die Identifizierbarkeit angegeben wird. Im Dänischen finden wir den Schlussartikel aber nur bei Skagenområdet und den vorangestellten Artikel (aufgrund des Adjektivs "årlig" (jährlich) bei den årlige Skagen Festival.

Bei Skagen by und bei St. Hans aften steht der 0-Artikel. Für diese Nominalphrasen gelten folgende pragmatische, morphologische und wortsemantische Bedingungen gleichzeitig: Der Gegenstand ist durch Allgemeinwissen identifizierbar, außerdem gilt die morphologische Bedingung, dass die Nominalphrase eine Art Apposition oder appositionelles Attribut enthält, und die wortsemantische Bedingung, dass es sich hierbei um einen Eigennamen handelt. In solchen Fällen steht im Dänischen meistens der 0-Artikel. Oft ist der Eigenname ein geographischer Name, z. B. Esbjerg Havn (der Hafen von Esbjerg).

Bei der Konstruktion mit Prädikativ En af dem, Anna Ancher, var Skagbo steht im Ausgangstext auch der 0-Artikel, was darauf hindeutet, dass die Zugehörigkeit zu einer Klasse angegeben werden soll.

\subsubsection{Artikelgebrauch im deutschen Zieltext}

Bei der Übersetzung von den årlige Skagen Festival in "das jährliche Skagen Festival" ist der Artikelgebrauch in beiden Sprachen gleich. Dasselbe gilt auch, wenn man Skagen-området mit "Das Gebiet Skagen" oder mit "Das Gebiet von Skagen" übersetzt. Benutzt man die nebengeordneten Nomina "Skagen und Umgebung" oder nur "Skagen", so ist der Artikelgebrauch 0-Artikel. Dagegen muss im Deutschen bei der Übersetzung von Skagen by und St. Hans aften/Sankthansaften der bestimmte Artikel verwendet werden.

Da beim Prädikatsnomen Skagbo, wie gesagt, von einer Klassenzugehörigkeit die Rede ist, wurde im Deutschen bei der Wahl einer Konstruktion mit Nomen oder Adjektiv + Nomen "(gebürtige/einheimische) Skagnerin" auch der 0-Artikel benutzt.

Im nachfolgenden Schema (Abb. 1) werden für den gegebenen Kontext die Übereinstimmungen und Unterschiede in der Struktur der Nominalphrasen und im Artikelgebrauch zusammengefasst: 


\begin{tabular}{|c|c|c|c|c|c|c|c|}
\hline 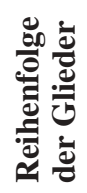 & 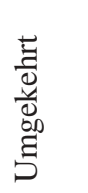 & 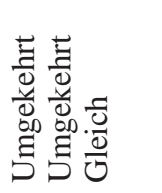 & $\frac{\frac{\pi}{0}}{\frac{0}{0}}$ & 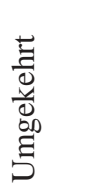 & & $\frac{\frac{\pi}{0}}{\frac{0}{0}}$ & $\frac{\bar{U}}{\frac{0}{0}}$ \\
\hline 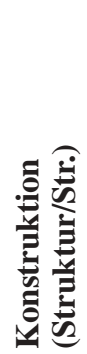 & 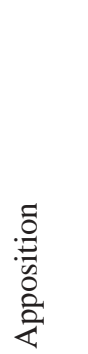 & 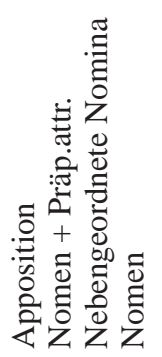 & 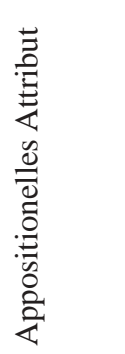 & 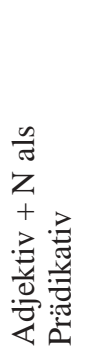 & 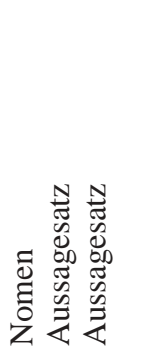 & 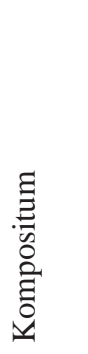 & 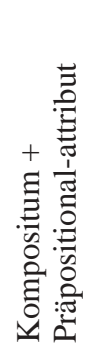 \\
\hline 焉 & $\begin{array}{l}\dot{E} \\
\dot{\vec{k}} \\
\dot{\vec{s}} \\
\infty\end{array}$ & 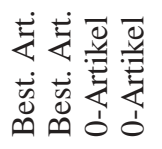 & $\begin{array}{l}\dot{\vec{z}} \\
\dot{\vec{\Delta}} \\
\dot{0}\end{array}$ & $\begin{array}{l}\bar{D} \\
\stackrel{y}{ \pm} \\
0 \\
0\end{array}$ & 离 & 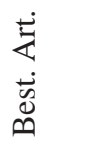 & $\begin{array}{l}\dot{\vec{z}} \\
\dot{\overrightarrow{0}} \\
\dot{0}\end{array}$ \\
\hline 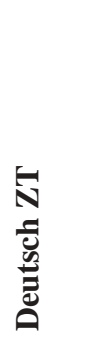 & 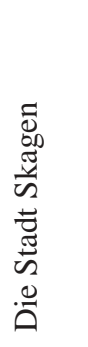 & 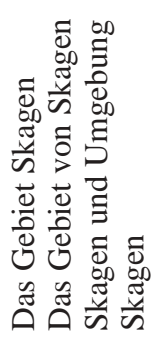 & 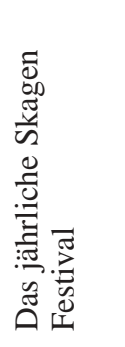 & 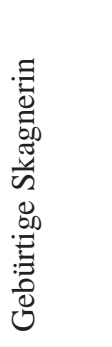 & 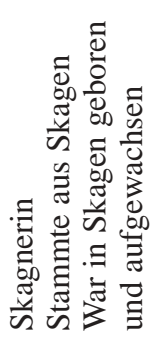 & 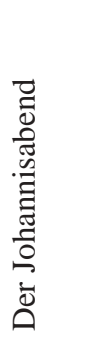 & 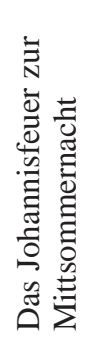 \\
\hline 焉 & 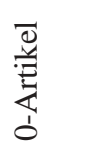 & 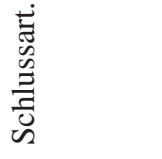 & $\begin{array}{l}\dot{\vec{z}} \\
\dot{\vec{n}} \\
\dot{0}\end{array}$ & 离 & & $\begin{array}{l}\overline{0} \\
\substack{0 \\
0 \\
0 \\
0}\end{array}$ & 离 \\
\hline 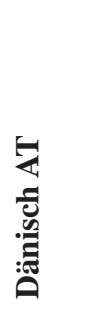 & 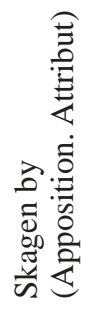 & 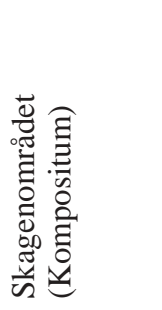 & 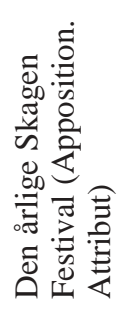 & \multicolumn{2}{|l|}{ 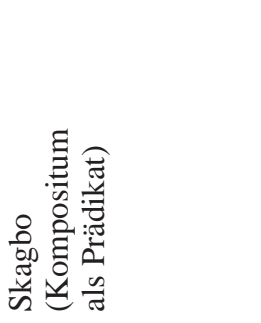 } & 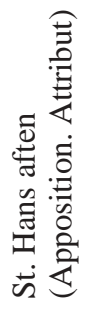 & 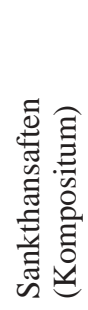 \\
\hline
\end{tabular}




\section{Ergebnisse der ÜmZ und der ÜoZ}

Im nachfolgenden Schema (Abb. 2) werden die Ergebnisse der beiden Übersetzungen, der ÜmZ und der ÜoZ, dargestellt. Die grau markierten Stellen zeigen die Fehler, die gemacht wurden, wobei in Klammern und kursiv die Art des Fehlers angegeben ist. Die Interferenzfehler sind entweder auf das direkte Überführen der dänischen Struktur zurückzuführen (Int.Str.), auf die direkte Übernahme des dänischen Artikelgebrauchs (Int.Art.), oder aber auf lexikalische Interferenz (Int.Lex). Das Problem der Interferenzphobie ist angegeben mit (Int.ph.). Es sind auch einige pragmatische (Pragm.) und semantische Probleme (Sem.) erwähnt, auf deren genauere Besprechung hier aber verzichtet wird. In der Spalte ganz rechts ist zu sehen, ob sich die Versuchsperson in der Übersetzung ohne Zeitdruck verbessern kann (VB), einen missglückten Versuch macht (MV), eine Verschlimmbesserung vornimmt (VS) oder das Problem ignoriert (IG).

\subsection{Kommentare}

In der ÜmZ wurden insgesamt 9 Interferenzfehler gemacht, bei denen die Struktur des AT direkt überführt wurde. Von diesen konnten in der ÜoZ nur 2 korrigiert werden (VB). Es wurden 6 Interferenzfehler beim Artikelgebrauch gemacht und von diesen konnten 3 korrigiert werden. Und es wurden 7 lexikalische Interferenzfehler gemacht, und von diesen konnten mit Hilfe der Wörterbücher 4 korrigiert werden. Ein neuer kam hinzu. Von den insgesamt 22 Interferenzfehlern wurden 9 korrigiert. Einer kam hinzu. Der Fehler, der aufgrund von Interferenzphobie entstanden ist, wurde nicht korrigiert.

Da es zur Vermeidung dieser Interferenzfehler nötig war, sowohl strukturelle als auch referenzielle und lexikalische Bedingungen zu beachten - in einigen Fällen Bedingungen aus allen drei Bereichen gleichzeitig ist es den Versuchspersonen selten gelungen, die fehlerhaften Vorschläge in der ÜmZ durch $100 \%$ ig korrekte Lösungen in der ÜoZ zu ersetzen. Eine Korrektur der gesamten Nominalphrase gelingt nur 2-mal, einmal bei Versuchsperson TRE, die "Skagen Stadt" richtig mit "Die Stadt Skagen" übersetzt, d.h. dass sowohl die Struktur als auch der Artikel korrigiert wird, und einmal bei Versuchsperson FEM, welche die prädikative Nominalphrase "Skagbo" durch den Aussagesatz "wurde hier geboren" umschreibt.

Nun könnte man vielleicht annehmen, dass die Versuchspersonen einfach an ihrer allerersten Lösung, an die sie sich bei der ÜoZ vielleicht noch erinnern können, festhalten. Es ist aber so, dass nur 17 von den 30 Nominalphrasen völlig gleich bleiben. 9-mal wird ein Fehler ignoriert (IG), 6-mal versuchen die Versuchspersonen doch etwas zu verbessern, was ihnen aber misslingt (MV), und 2-mal haben sie in der ÜmZ etwas richtig gemacht, was sie in der ÜoZ dann verwerfen (VS).

Es zeigt sich bei diesen Fehlern ein erstaunlich geringes Verbesserungsvermögen zwischen der ÜmZ und der ÜoZ, was auf ein Fehlen an Regeln, Methoden und Strategien hindeutet. 


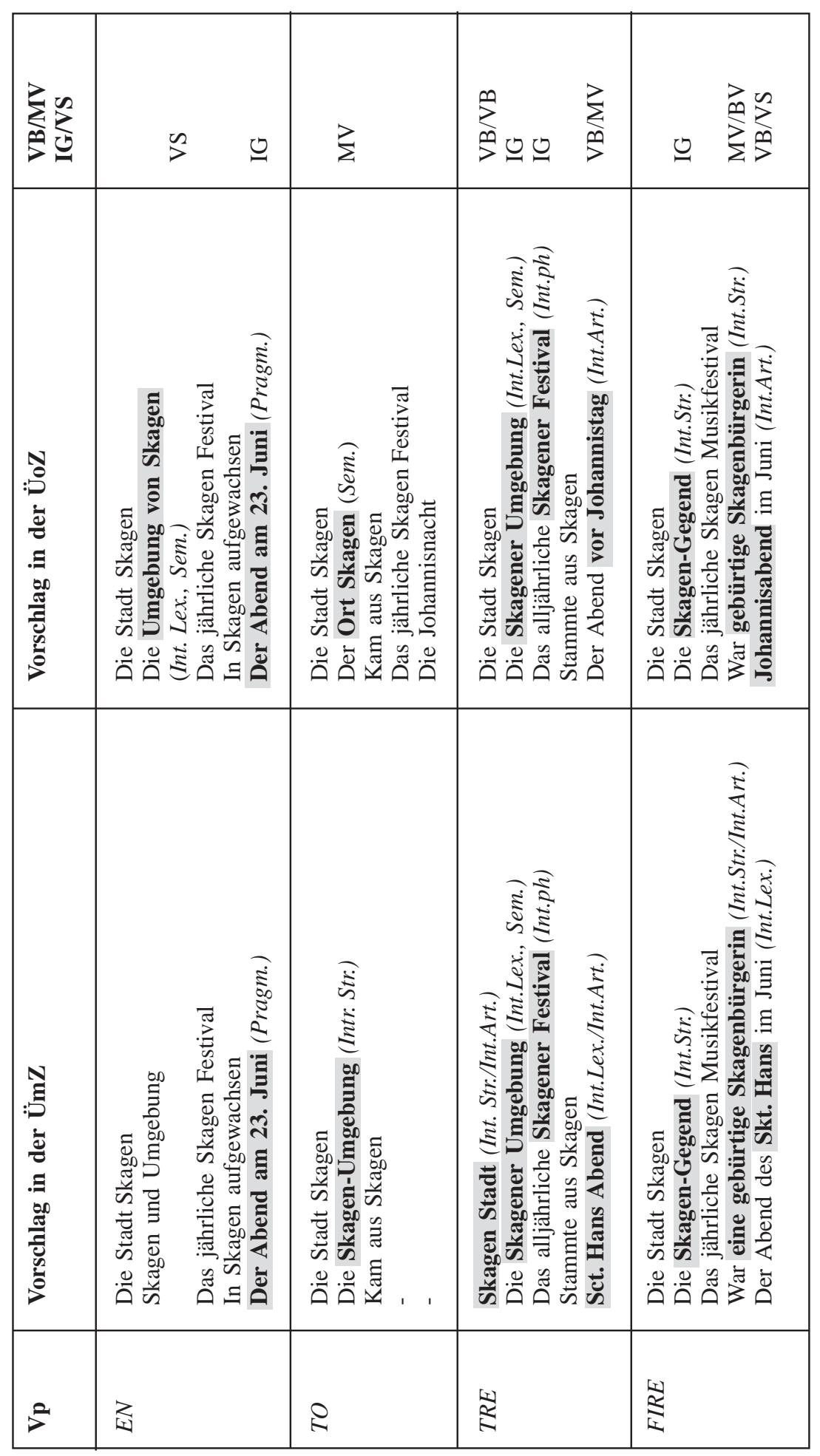




\begin{tabular}{|c|c|c|}
\hline$\sum_{i=0}^{\infty}$ & $\sum_{\Sigma}^{\infty} \quad \sum_{\infty}^{\infty} \sum_{>}^{\infty}$ & @ \\
\hline 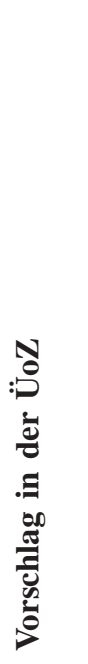 & 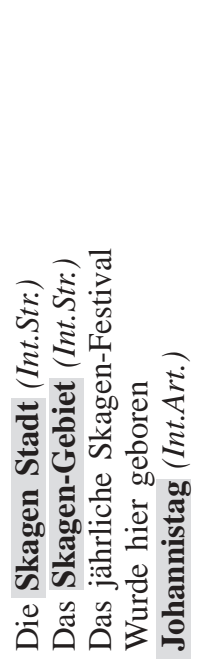 & $\begin{array}{ll} & \\
0\end{array}$ \\
\hline 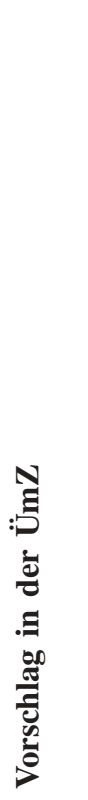 & 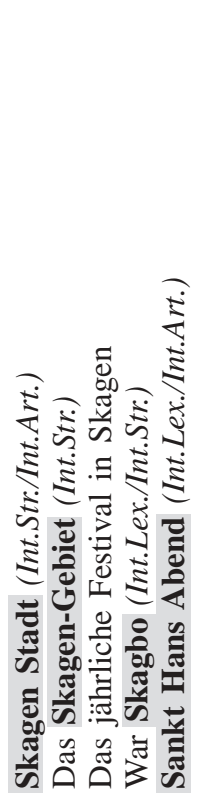 & 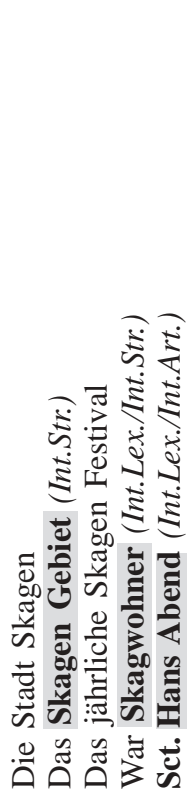 \\
\hline$c^{2}$ & $\underset{\substack{x|1\\
|}}{i}$ & $\begin{array}{l}\sqrt{2} \\
\sqrt[1]{2}\end{array}$ \\
\hline
\end{tabular}




\subsection{Der Übersetzungsprozess}

Um festzustellen, ob die Versuchspersonen an den problematischen Textstellen nachgedacht hatten, untersuchte ich zuerst ihren Zeitverbrauch unmittelbar im Zusammenhang mit den Interferenzfehlern. Dieser wird in der Tabelle der Abb. 4 als Zeit in Sek. für die ÜmZ und für die ÜoZ angegeben. Danach überprüfte ich bei der Retrospektion mit Replay, ob sie sich an den entsprechenden Textstellen unaufgefordert zu ihren Fehlern äußerten, d.h. ob sie sich der Probleme überhaupt bewusst waren. Dieses wird durch + (für ja) und - (für nein) angegeben. Schließlich registrierte ich in dem Interview, ob sie über ihre Probleme sprechen konnten und inwieweit sie automatisch, d.h. "nach Gefühl” übersetzt hatten oder bewusst. Das Ergebnis steht in der Tabelle unter Autom./Bewusst: A für automatisch und B für bewusst.

\subsubsection{Zeitverbrauch}

Es zeigte sich, dass die Versuchspersonen in der ÜoZ häufiger (10-mal) länger über die richtig übersetzten Textstellen nachdachten, die sie auch schon in der ÜmZ richtig übersetzt hatten, als über die in beiden Übersetzungen fehlerhaften Textstellen (6-mal), vgl. Abb. 4.

An 9 der 15 in der ÜoZ fehlerhaften Stellen verwendeten sie für die ÜoZ nicht mehr Zeit als für die ÜmZ. Daraus lässt sich schließen, dass ihnen ihre Probleme kaum aufgefallen sind.

\subsubsection{Retrospektion und Interview im Verhältnis zum Zeitverbrauch}

Die Versuchsperson SEKS bemerkt während der Retrospektion kein einziges ihrer Probleme und übersetzt automatisch. Aus dem Interview geht hervor, dass sie, auch wenn sie direkt auf ein Problem aufmerksam gemacht wird, nichts dazu sagen kann, weil ihr das elementare grammatische Wissen und die Terminologie fehlt. Und ihr Zeitverbrauch zeigt, dass sie auf die fehlerhaften Textstellen auch keine Zeit verwendet. Versuchsperson TO sagt auch nicht viel, bemerkt das eine Problem nicht und kann im Interview auch nichts dazu sagen. An drei anderen Textstellen übersetzt sie bewusst richtig. Versuchsperson FIRE bemerkt während der Retrospektion auch eines ihrer drei Probleme nicht und kann im Interview zu zwei Problemen nichts sagen. Sie übersetzt automatisch, verwendet in der ÜoZ kaum Zeit auf ihre Probleme und kann kaum etwas daran verbessern. Versuchsperson FEM erwähnt alle ihre Probleme unaufgefordert und ist in zwei Fällen auch fähig, das Problem zu erklären, in einem Fall nicht. Sie benutzt auch Zeit zur Lösung. Aus dem Interview geht aber hervor, dass sie ihre Übersetzungsvorschläge trotz aller Kommentare ganz willkürlich und nach Gefühl einsetzt und dann doch Fehler macht. Die Versuchspersonen EN und TRE erwähnen während der Retrospektion nur ein Problem unaufgefordert und übersetzen alle anderen Stellen automatisch. 
Im Zusammenhang mit der Nominalphrase "St. Hans aften” erwähnen während der Retrospektion 5 der Versuchspersonen das Problem unaufgefordert. Sie sind sich auch im Interview dessen bewusst, lösen können sie es aber trotz Wörterbuch und Internet nicht.

Man kann also auch aus der Retrospektion und den Interviews ableiten, dass die Versuchspersonen diesen Interferenzproblemen hilflos gegenüberstehen. Dabei handelt es sich gar nicht um unwesentliche Unterschiede zwischen den beiden Sprachen. Besonders in Fachtexten, z. B. in juristischen und technischen Texten treten gerade diese Probleme sehr häufig auf (Hansen 1995).

Alle Versuchspersonen geben vor allem Kommentare zu den lexikalischen Problemen ab, d.h. besonders zu ihrer Suche in Wörterbüchern oder im Internet. Der strukturellen Unterschiede (das häufigste Problem) sind sie sich überhaupt nicht bewusst, und zum Artikelgebrauch können sie auch nicht viel sagen. Nur Versuchsperson FEM erwähnt den Artikelgebrauch mit einer sehr allgemeinen Bemerkung:

Jetzt müssen noch Artikel eingesetzt werden - die nehme ich sehr ernst. Ich passe sehr darauf auf, im Deutschen immer einen Artikel zu haben - sie haben fast immer Artikel, wo wir im Dänischen oft keinen haben.

\subsection{Kontrollversuch: ein anderes komplexes Problem}

Aufgrund dieses Ergebnisses wurde das Verbesserungsvermögen im Zusammenhang mit einem anderen komplexen Interferenzproblem untersucht, das auch im AT vorkam. Ich wollte überprüfen, ob die Versuchspersonen ganz grundsätzlich keine Verbesserungsfähigkeit besaßen, oder ob das nur bei den erwähnten erweiterten Nominalphrasen der Fall war. Das Beispiel ist:

( ) Gennem generationer har folk fra nær og fjern valfartet til dette eftertragtede sted på Danmarks nordligste punkt. Die wörtliche Übersetzung ist:

*Durch Generationen sind die Leute von nah und fern zu diesem angestrebten Ort auf Dänemarks nördlichstem Punkt gewallfahrtet. Besser:

Seit Generationen wallfahren Menschen aus nah und fern an diesen beliebten Ort an der Nordspitze Dänemarks.

Die typischen Fehler sind markiert. Sie entstehen einerseits durch die direkte Überführung der Präposition und anderseits durch die Übernahme der Zeit: Perfekt statt Präsens.

Vergleicht man für diese Textstelle die Vorschläge der ÜmZ mit denen der ÜoZ (siehe Abb. 3), so stellt man fest, dass die Verbesserungsfähigkeit der Versuchspersonen hier viel größer ist: 3 der Versuchspersonen können beide Interferenzfehler vermeiden. Eine hat sie schon in der ÜmZ nicht gemacht. Von insgesamt 10 Fehlern in der ÜmZ sind 6 in der ÜoZ korrigiert 
worden. Das Verbesserungsvermögen ist im Hinblick auf diese Fehler wesentlich größer.

Die Retrospektion zeigt, dass 5 von 6 Versuchspersonen das Problem unaufgefordert kommentieren. Der Zeitverbrauch zeigt, dass 4 der Versuchspersonen in der ÜoZ lange über die Textstelle nachdenken (über eine Minute). Nur 2 der Versuchspersonen wirken hilflos (TO und SEKS). Bei diesem Problem scheinen die Versuchspersonen also wesentlich bessere Voraussetzungen mitzubringen, was nicht verwundert, denn es wird im Grammatik- und Übersetzungsunterricht häufig behandelt und es wird in den gebräuchlichen Grammatiken (z. B. Bruun Hansen 2002:68) kontrastiv erwähnt. 


\begin{tabular}{|c|c|c|c|c|c|c|}
\hline$\sum_{i=0}^{i}$ & $\sum_{>}^{\infty}$ & $\stackrel{\circlearrowright}{\circlearrowright}$ & $\sum_{>}^{\infty}$ & $\sum_{>}^{\infty}$ & $\frac{\ddot{1}}{0}$ & $\stackrel{\circlearrowright}{\stackrel{0}{O}}$ \\
\hline 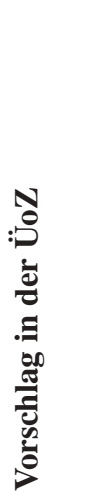 & 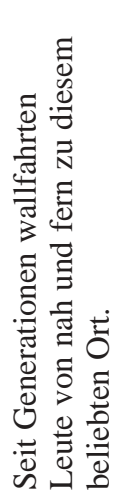 & 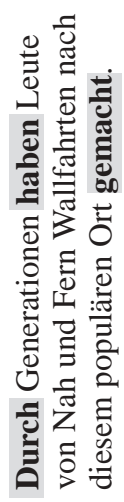 & 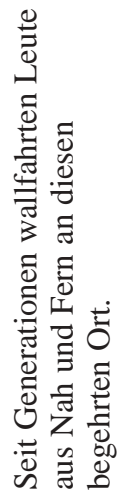 & 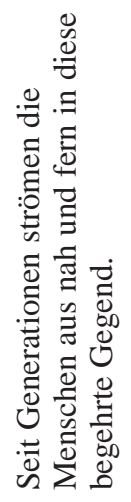 & 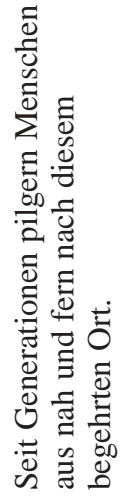 & 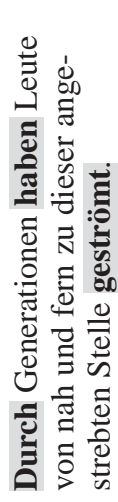 \\
\hline 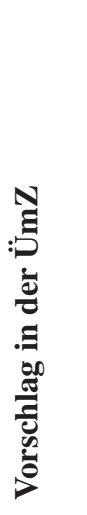 & 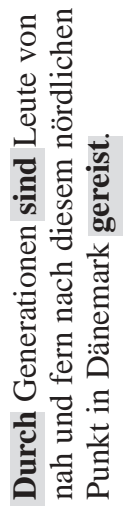 & 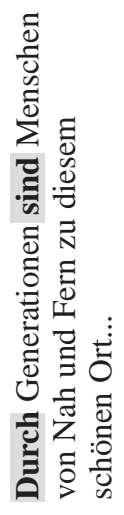 & 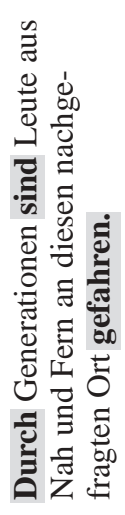 & 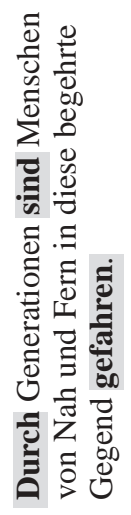 & 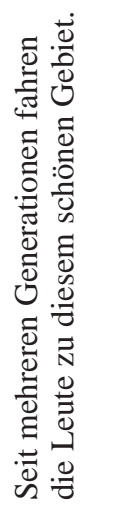 & 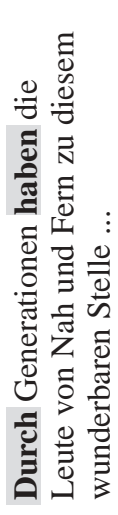 \\
\hline 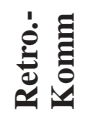 & + & + & + & ' & + & + \\
\hline \multirow{2}{*}{ 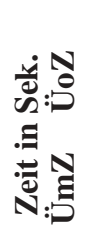 } & $\begin{array}{l}8 \\
\stackrel{8}{0} \\
\stackrel{-}{-}\end{array}$ & $\begin{array}{l}\tilde{n} \\
\dot{ \pm} \\
=\end{array}$ & 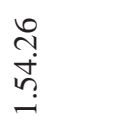 & 0 & $=$ & 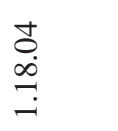 \\
\hline & $m$ & ㄷ & $\infty$ & 0 & $r$ & $\infty$ \\
\hline$\vec{p}^{2}$ & 过 & 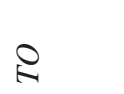 & 닌 & $\underset{1}{2}$ & 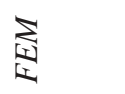 & 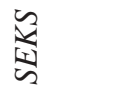 \\
\hline
\end{tabular}




\begin{tabular}{|c|c|c|c|c|}
\hline$\sum_{i=0}^{\infty}$ & $\stackrel{0}{>}$ & $z$ & $\sum_{\substack{\infty \\
\infty}}^{\infty} \sum_{\sum^{\prime}}^{z}$ & $0 \sum_{\sum}^{\infty} \sum_{\infty}^{\infty}$ \\
\hline 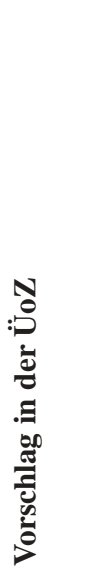 & 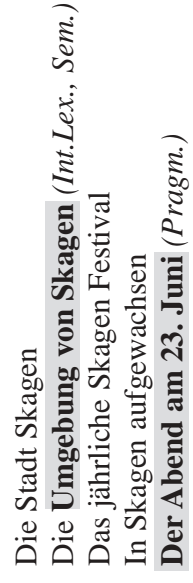 & 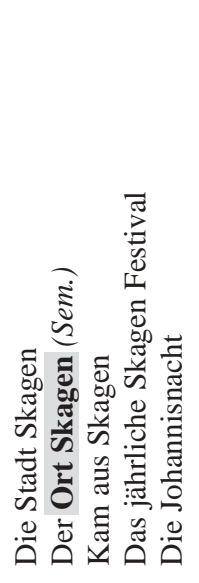 & 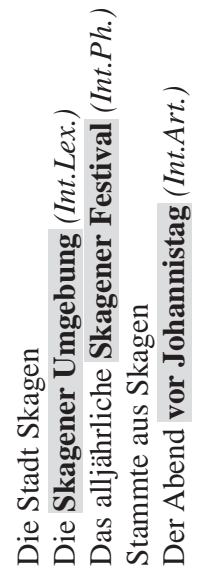 & 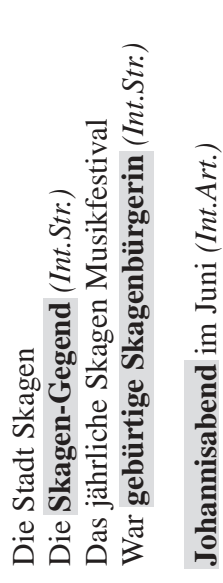 \\
\hline 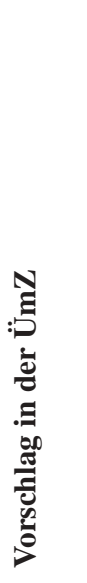 & 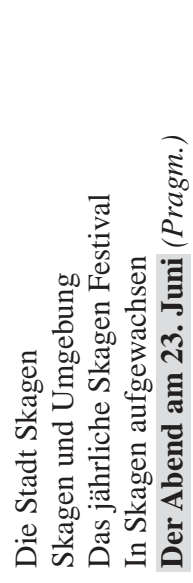 & 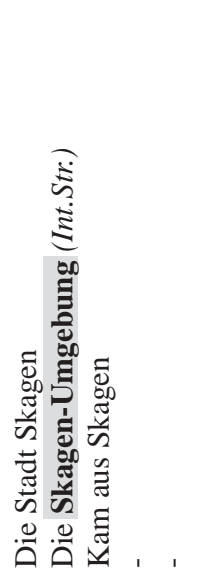 & 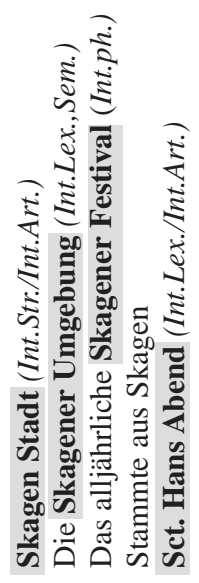 & 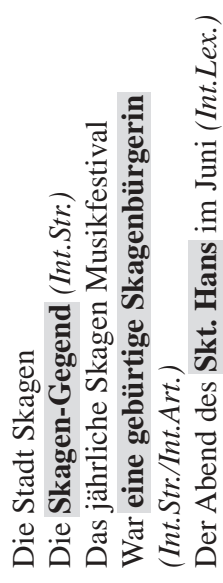 \\
\hline 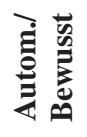 & $\ll \ll \ll \ll \oplus$ & $\ll \ll \curvearrowleft ص \curvearrowleft$ & $\ll \ll \ll \ll \varphi$ & $\ll \ll \ll \ll$ \\
\hline 它 & $1 \quad 1 \quad 1 \quad 1+$ & $1 \quad 1 \quad 1 \quad 1+$ & $1 \quad 1 \quad 1 \quad 1 \quad+$ & $1,++$ \\
\hline \multirow{2}{*}{ 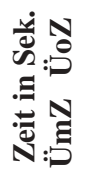 } & $\stackrel{\infty}{+}-\stackrel{\sim}{\sim} \sigma \stackrel{\circ}{n}$ & $=\cong \bullet I \infty$ & 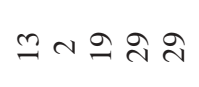 & no- \\
\hline & $\approx 0 n m=$ & $=\subseteq N \quad$. & $\because+O \underline{m}$ & 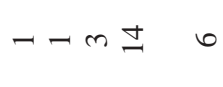 \\
\hline$z^{2}$ & 诖 & $R$ & $\frac{1}{k}$ & $\underset{2}{2}$ \\
\hline
\end{tabular}




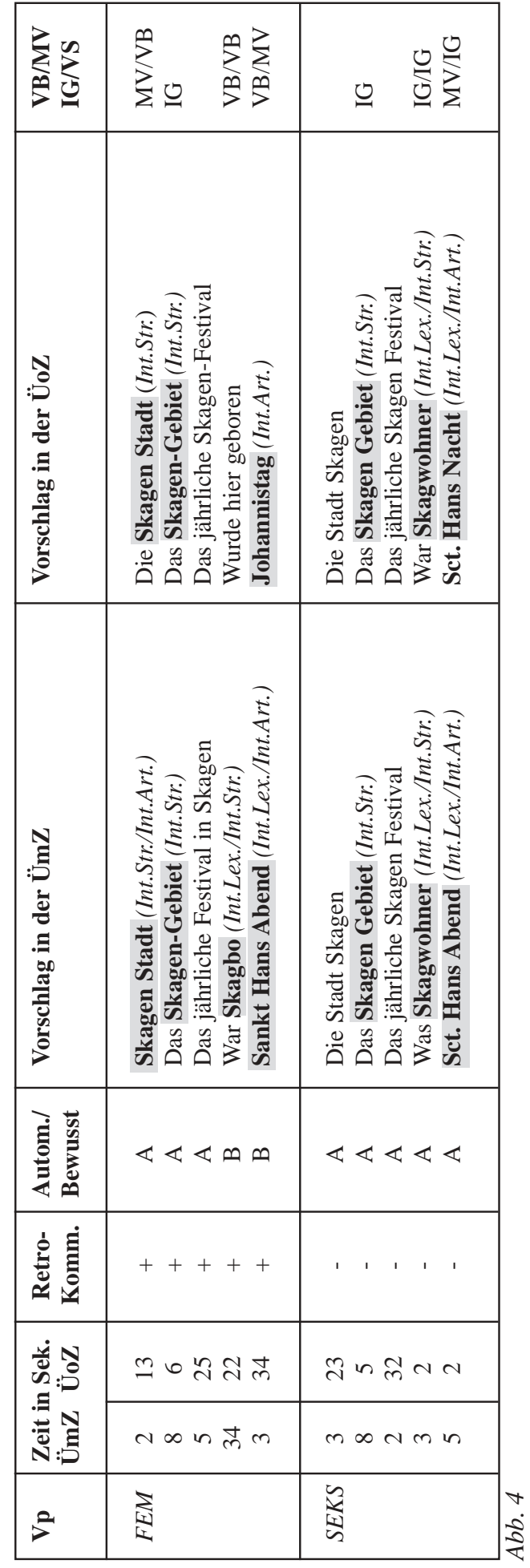




\section{Konklusion}

Innerhalb ein und derselben Sprache ist es oft möglich, verschiedene Attribute, die eine ähnliche semantische Funktion haben, oder unterschiedlich strukturierte Nominalphrasen ohne große Bedeutungsunterschiede auszutauschen, z. B. ein Kompositum gegen eine Nominalphrase mit einem Adjektiv oder gegen eine Nominalphrase mit einem appositionellen Attribut und umgekehrt. In einem Kontext ist man aber meistens nicht im Zweifel darüber, welche der Formen vorzuziehen ist, und wann bei Austausch der Konstruktionen ein Bedeutungsunterschied entstehen würde (Hansen 1995:38).

Beim Übersetzen kann man nicht davon ausgehen, dass in beiden Sprachen identisch strukturierte Nominalphrasen benutzt werden. Man kann nicht einmal immer damit rechnen, dass in beiden Sprachen überhaupt Nominalphrasen benutzt werden (Hansen 1986:76ff.). Daher ist es wichtig, dass auf die in der Zielsprache vorhandenen Möglichkeiten aufmerksam gemacht wird, denn nur dann kann die Übersetzerin oder der Übersetzer darauf achten und entscheiden, welcher in einer gegebenen Situation der treffendste Ausdruck ist.

Die Versuche haben gezeigt, dass es Probleme, wie z. B. das der Interferenz bei Referenz gibt, mit dem semiprofessionelle Übersetzerinnen und Übersetzer allein kaum fertig werden, weil sie die Konstruktionen oder Teile davon nicht durchschauen, und weil sie den Unterschieden keine Beachtung schenken. Es reicht nicht aus, dass man, wie z. B. die Versuchsperson FEM, mit leichter Hand Artikel über die Nominalphrasen des deutschen Textes streut - wenn man nicht auch die unterschiedlichen Strukturen erkannt hat.

Weder die Beschreibungen in Grammatiken einzelner Sprachen, noch groß angelegte kontrastive Analysen helfen den Übersetzerinnen und Übersetzern viel, wenn solche Probleme schnell gelöst werden sollen.

Was sie dringend brauchen könnten, wären benutzerfreundliche Beschreibungen, die in übersichtlicher Form zeigen, wie das, was in einer Sprache in einem Text mit einer bestimmten typischen Funktion in einer bestimmten typischen Kommunikationssituation normalerweise durch eine bestimmte sprachliche Form ausgedrückt wird, wenn/weil dort die semantischen, syntaktischen, morphologischen, phonologischen, stilistischen Bedingungen: $\beta, \mathrm{v}, \mathrm{x}, \mathrm{y}, \mathrm{z}$ gelten, in der anderen Sprache unter den dort geltenden Bedingungen: $\mathrm{x}, \mathrm{y}, \mathrm{z}, \mathfrak{x}, \emptyset$ ausgedrückt werden kann oder soll. Bei jedem Problemkomplex wäre zu überlegen, welche Strategien man aufgrund der Unterschiede zwischen den Sprachen beim Hinübersetzen oder beim Herübersetzen beachten und anwenden müsste. Die kontrastive Linguistik könnte einen wichtigen Beitrag leisten, indem sie vor dem Hintergrund ihrer einzelsprachlichen und kontrastiven Analysen, kurze, überschaubare benutzerfreundliche Gebrauchsregeln zu von der Übersetzungswissenschaft ausgewählten Themen und Problemen anbieten würde. Eine engere $\mathrm{Zu}$ sammenarbeit zwischen Linguisten und Übersetzern könnte hier viel leisten. Der Beitrag der Übersetzungswissenschaft zur Linguistik wäre, dass sie 
auf Problemfelder wie die in diesem Artikel beschriebenen aufmerksam macht.

\section{Bibliographie}

Bruun Hansen, Agnete, Eriksen, Carl Collin \& Elva Senestad (22002). Gyldendal Tysk Grammatik. Kopenhagen: Gyldendal.

Diderichsen, Paul ( $\left.{ }^{3} 1970\right)$. Elementor dansk grammatik. Kopenhagen: Gyldendal.

Fabricius-Hansen, Cathrine \& Arnim von Stechow (1989). "Explikative und implikative Nominalerweiterungen im Deutschen." Zeitschrift für Sprachwissenschaft 8, 173-205.

Hansen, Aage (1967). Moderne Dansk. Kopenhagen: Grafisk Forlag.

Hansen, Gyde (1986). Kontrastive Analyse des Artikelgebrauchs im Dänischen und Deutschen. Kopenhagen: Nyt Nordisk Forlag Arnold Busck.

Hansen, Gyde (1995). Kontrastive Textgrammatik. Kopenhagen: Samfundslitteratur.

Hansen, Gyde (1997). "Habacht-Signale beim Übersetzen in die Fremdsprache (am Beispiel Dänisch-Deutsch)." Eberhard Fleischmann, Wladimir Kutz \& Peter A. Schmitt (Hrsg.) (1997). Translationsdidaktik. Tübingen: Gunter Narr, 133-139.

Hansen, Gyde (1998). "Studies in article usage in Danish and German." Gyde Hansen (Hrsg.) (1998). Determination. Copenhagen Studies in Language (CSL) 21. Kopenhagen: Samfundslitteratur, 9-66.

Hansen, Gyde (1999). "Das kritische Bewusstsein beim Übersetzen." Gyde Hansen (Hrsg.) (1999). Probing the Process in Translation: Methods and Results. CSL 24. Kopenhagen: Samfundslitteratur, 43-67.

Hansen, Gyde (2002). "Selbstaufmerksamkeit im Übersetzungsprozess." Gyde Hansen (Hrsg.) (2002). Empirical Translation Studies: Process and Product. CSL 27. Kopenhagen: Samfundslitteratur, 9-27.

Heger, Klaus (1971). Monem, Wort und Satz. Tübingen: Niemeyer.

Jakobsen, Arnt Lykke (1999a). "Logging target text production with Translog." Gyde Hansen (Hrsg.) (1999). Probing the Process in Translation: Methods and results. CSL 24. Kopenhagen: Samfundslitteratur, 9-20.

Jakobsen, Arnt Lykke (1999b). "Translog documentation." Gyde Hansen (Hrsg.) (1999). Probing the Process in Translation: Methods and Results. CSL 24. Kopenhagen: Samfundslitteratur, 149-184.

Juhász, János (1970). Probleme der Interferenz. München: Max Hueber.

Kupsch-Losereit, Sigrid (1998). "Interferenzen.” Mary Snell-Hornby et al. (Hrsg.) (1998). Handbuch Translation. Tübingen: Stauffenburg, 167-170.

Kussmaul, Paul (1995). Training the Translator. Amsterdam/Philadelphia: Benjamins.

Mikkelsen, Kristian (1911/21975). Dansk Ordføjningslaere. Kopenhagen: Hans Reitzel.

Ogden, Charles K. \& Ivor Armstrong Richards (1923). The Meaning of Meaning. London: Routledge \& Kegan Paul.

Schreiber, Michael (in Druck). "Kontrastive Linguistik und sprachenpaarbezogene Translationswissenschaft.“. Daniel Gile, Gyde Hansen \& Kirsten Malmkjær (Hrsg.). Claims, Changes and Challenges in Translation Studies. Selected Contributions From the EST Congress, Copenhagen 2001. Amsterdam/Philadelphia: Benjamins.

Snell-Hornby, Mary (1986). Übersetzungswissenschaft. Eine Neuorientierung. Tübingen: Francke. 
Strawson, Peter Frederik (1950). “On Referring.” Mind 59, 320-344.

Vater, Heinz (1963/21979). Das System der Artikelformen im gegenwärtigen Deutsch. Tübingen: Niemeyer.

Wilss, Wolfram (1994). "Kontrastive Linguistik und Übersetzungswissenschaft Versuch einer Abgrenzung." Deutsch als Fremdsprache 1, 13-19.

\footnotetext{
${ }^{1}$ Je nachdem, welche Anschauung man im Hinblick auf Nullstellen im Paradigma der Artikel und Pronomina mit derselben Funktion hat, kann man von "keinem" Artikel oder vom "0-Artikel" sprechen (Hansen 1983:9 und 1998:22). Ich werde hier „0-Artikel“" benutzen.
} 


\section{Anhang}

Übersetzungsauftrag: Turistbrochuren "Lyset over Skagen” skal også trykkes på tysk. Du bliver bedt om at oversætte følgende afsnit:

(Die Broschüre "Lyset over Skagen” soll auch auf Deutsch gedruckt werden. Man bittet Sie, folgende Abschnitte zu übersetzen.)

\begin{tabular}{|c|c|}
\hline Ausgangstext & $\begin{array}{l}\text { Zieltext (unverändert aus der } \\
\text { Broschüre) }\end{array}$ \\
\hline $\begin{array}{l}\text { Se lyset. Nyd naturen og havet. } \\
\text { Mærk miljøet... } \\
\text { Det første man lægger mærke til, } \\
\text { når man nærmer sig Skagen, er det } \\
\text { vidunderlige, stærke lys. Dernæst } \\
\text { en natur så storslået, vidtstrakt og } \\
\text { uspoleret med skov, klitter og he- } \\
\text { dearealer omkranset af hav. Det } \\
\text { milde børnevenlige Kattegat og det } \\
\text { mere barske Vesterhav. Her lever } \\
\text { menneskene af havet og med natu- } \\
\text { ren i et rent og sundt miljø. Gennem } \\
\text { generationer har folk fra nor og } \\
\text { fjern valfartet til dette eftertragtede } \\
\text { sted på Danmarks nordligste punkt, } \\
\text { hvor solen skinner mest i hele lan- } \\
\text { det. Her mødes man året rundt, for- } \\
\text { di stedet er noget ganske særligt. }\end{array}$ & $\begin{array}{l}\text { Das Licht erleben. Natur und } \\
\text { Meer als intakte Umwelt ge- } \\
\text { nießen... } \\
\text { Wenn man sich Skagen nähert, } \\
\text { bemerkt man zuerst das wunder- } \\
\text { bare, starke Licht - dann die } \\
\text { großartige, weiträumige und intakte } \\
\text { Naturlandschaft mit Wald, Dünen } \\
\text { und Heide. Meerumschlungen zwi- } \\
\text { schen dem milden und kinder- } \\
\text { freundlichen Kattegat-Strand und } \\
\text { der eher rauhen Nordsee-Küste. } \\
\text { Hier leben die Menschen in } \\
\text { Eintracht von und mit dem Meer } \\
\text { und eine heile Umwelt, die die } \\
\text { Lebensgrundlage der Einheimi- } \\
\text { schen ist. Seit Generationen pilgern } \\
\text { Menschen aus nah und fern zu } \\
\text { diesem attraktiven Ort hoch im } \\
\text { Norden Dänemarks, wo die Sonne } \\
\text { am meisten scheint. Hier trifft man } \\
\text { sich das ganze Jahr, denn der Ort } \\
\text { hat was Besonderes. }\end{array}$ \\
\hline $\begin{array}{l}\text { Malerne var de første turister... } \\
\text { Skagen by, der som købstad er ca. } \\
600 \text { år gammel, har et berømt miljø } \\
\text { med en spændende historie. Malet } \\
\text { og beskrevet af kunstnere netop på } \\
\text { grund af det specielle lys, de gule } \\
\text { huse med røde tegltage og hvide } \\
\text { blonder, naturen og fiskernes liv og } \\
\text { færden. Det hele begyndte midt i } \\
\text { 1800-tallet, og malere som P.S. } \\
\text { Krøyer, Anna und Michael Ancher, } \\
\text { digterern og maleren Holger Drach- }\end{array}$ & $\begin{array}{l}\text { Die Maler waren die ersten Tou- } \\
\text { risten... } \\
\text { Die Stadt Skagen, der die Stadt- } \\
\text { rechte vor ca. } 600 \text { Jahren verliehen } \\
\text { wurde, ist eine Gemeinde mit einer } \\
\text { interessanten Geschichte. Die Stadt } \\
\text { wurde gerade wegen ihres beson- } \\
\text { deren Lichts, der gelben Häuser mit } \\
\text { roten Ziegeldächern und weißen } \\
\text { Blonden, der Natur und des bunten } \\
\text { Treibens der Fischer von Künstlern } \\
\text { gemalt und beschrieben. Es begann } \\
\text { alles in der Mitte des vorigen Jahr- }\end{array}$ \\
\hline
\end{tabular}




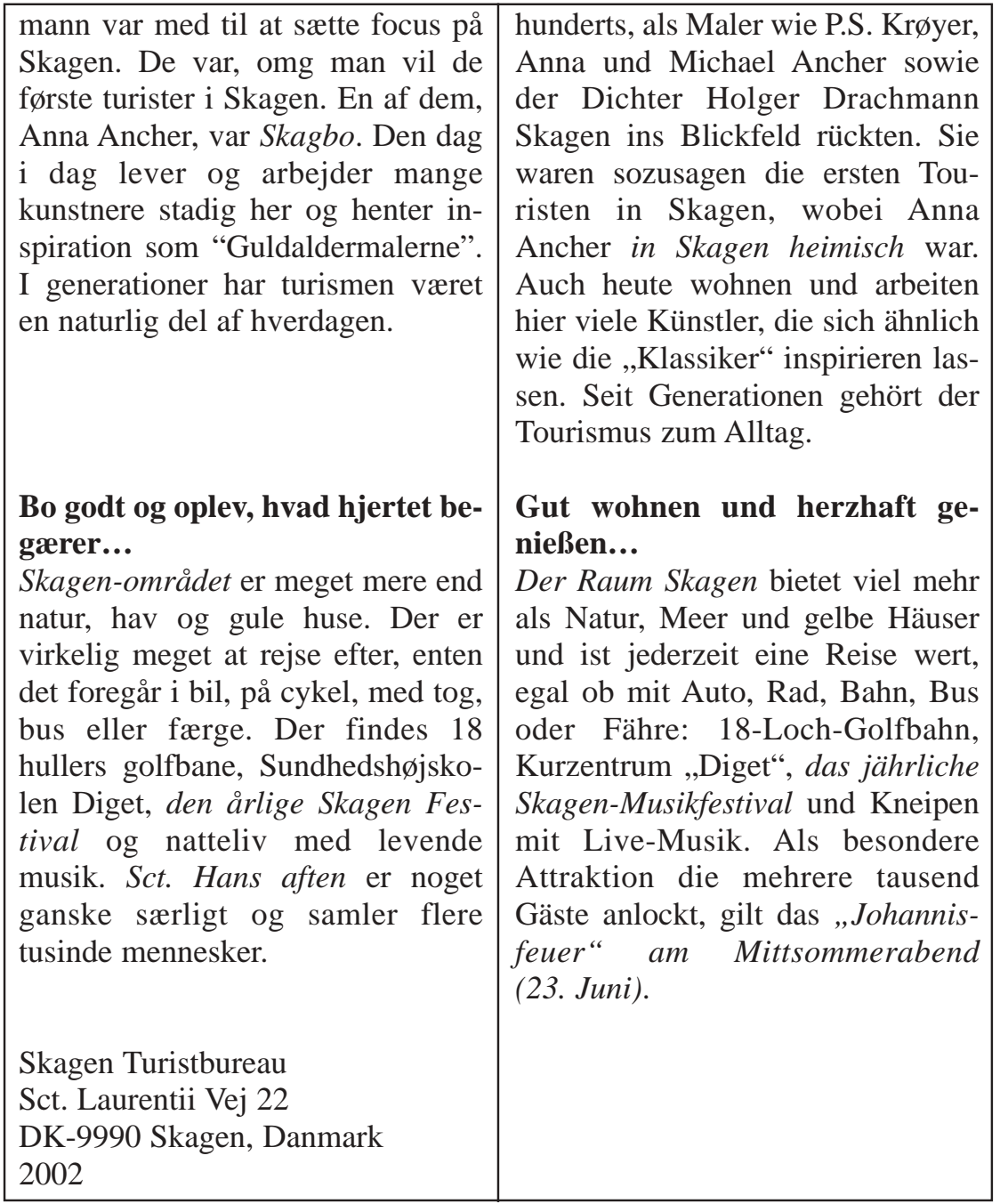

